\title{
Multiregion gene expression profiling reveals heterogeneity in molecular subtypes and immunotherapy response signatures in lung cancer
}

\author{
Won-Chul Lee ${ }^{1,2} \cdot$ Lixia Diao $^{3} \cdot$ Jing Wang ${ }^{3} \cdot$ Jianhua Zhang $^{1} \cdot$ Emily B. Roarty ${ }^{2}$ Susan Varghese ${ }^{2} \cdot$ Chi-Wan Chow $^{4}$. \\ Junya Fujimoto ${ }^{4}$ Carmen Behrens ${ }^{2} \cdot$ Tina Cascone $^{2} \cdot$ Weiyi Peng $^{5} \cdot$ Neda Kalhor $^{6} \cdot$ Cesar A. Moran $^{6}$. \\ Annikka Weissferdt ${ }^{6} \cdot$ Faye M. Johnson ${ }^{2} \cdot$ William N. William Jr. $^{2} \cdot$ Stephen G. Swisher ${ }^{7} \cdot$ J. Jack Lee (iD) ${ }^{8}$. \\ Waun Ki Hong ${ }^{2} \cdot$ John V. Heymach ${ }^{2} \cdot$ Ignacio I. Wistuba $^{2,4} \cdot$ P. Andrew Futreal ${ }^{1}$ Jianjun Zhang ${ }^{1,2}$
}

Received: 25 October 2017 / Revised: 7 December 2017 / Accepted: 10 December 2017 / Published online: 6 February 2018

(c) United States \& Canadian Academy of Pathology 2018

\begin{abstract}
Intra-tumor heterogeneity may be present at all molecular levels. Genomic intra-tumor heterogeneity at the exome level has been reported in many cancer types, but comprehensive gene expression intra-tumor heterogeneity has not been well studied. Here, we delineated the gene expression intra-tumor heterogeneity by exploring gene expression profiles of 35 tumor regions from 10 non-small cell lung cancer tumors (three or four regions/tumor), including adenocarcinoma, squamous cell carcinoma, large-cell carcinoma, and pleomorphic carcinoma of the lung. Using Affymetrix Gene 1.0 ST arrays, we generated the gene expression data for every sample. Inter-tumor heterogeneity was generally higher than intra-tumor heterogeneity, but some tumors showed a substantial level of intra-tumor heterogeneity. The analysis of various clinically relevant gene expression signatures including molecular subtype, epithelial-to-mesenchymal transition, and anti-PD-1 resistance signatures also revealed heterogeneity between different regions of the same tumor. The gene expression intratumor heterogeneity we observed was associated with heterogeneous tumor microenvironments represented by stromal and immune cells infiltrated. Our data suggest that RNA-based prognostic or predictive molecular tests should be carefully conducted in consideration of the gene expression intra-tumor heterogeneity.
\end{abstract}

These authors contributed equally: Won-Chul Lee, Lixia Diao, Jing Wang.

Electronic supplementary material The online version of this article (https://doi.org/10.1038/s41379-018-0029-3) contains supplementary material, which is available to authorized users.

$\triangle$ Ignacio I. Wistuba

iiwistuba@mdanderson.org

$\triangle$ P. Andrew Futreal

afutreal@mdanderson.org

$\triangle$ Jianjun Zhang

jzhang20@mdanderson.org

1 Department of Genomic Medicine, The University of Texas MD Anderson Cancer Center, Houston, TX, USA

2 Department of Thoracic/Head and Neck Medical Oncology, The University of Texas MD Anderson Cancer Center, Houston, TX, USA

3 Department of Bioinformatics and Computational Biology, The
Cancers are heterogeneous diseases. Substantial inter-tumor heterogeneity of many cancers has been well documented [1]. Heterogeneity also exists within individual tumors, as a single tumor can display multiple different molecular features within it, a phenomenon termed intra-tumor heterogeneity. Genomic intra-tumor heterogeneity has been

University of Texas MD Anderson Cancer Center, Houston, TX, USA

4 Department of Translational Molecular Pathology, The University of Texas MD Anderson Cancer Center, Houston, TX, USA

5 Department of Melanoma Medical Oncology, The University of Texas MD Anderson Cancer Center, Houston, TX, USA

6 Department of Pathology, The University of Texas MD Anderson Cancer Center, Houston, TX, USA

7 Department of Thoracic Surgery, The University of Texas MD Anderson Cancer Center, Houston, TX, USA

8 Department of Biostatistics, The University of Texas MD Anderson Cancer Center, Houston, TX, USA 
reported in both hematologic and solid tumors and may be associated with treatment failure and emergence of drug resistance [2]. Gerlinger and colleagues [3, 4] demonstrated profound genomic intra-tumor heterogeneity in renal cell carcinomas using a multiregion whole-exome sequencing approach. Using similar approach, our group [5] and de Bruin et al. [6] have independently shown clear evidence of genomic intra-tumor heterogeneity of non-small cell lung cancers, but to a much less extent compared to that in renal cell carcinomas, suggesting different cancers may have different genomic intra-tumor heterogeneity architecture.

RNA and protein expression intra-tumor heterogeneity has also been studied, but only at single gene and limited gene panel levels. For example, spatial heterogeneity of estrogen receptor and human epidermal growth factor receptor 2 (HER-2) protein was reported in breast cancers [7, 8] and HER-2 protein expression was found to be discordant between different regions within the same gastric cancers [9]. Gyanchandani and colleagues [10] analyzed five RNA expression-based prognostic panels on 181 tumor samples from 71 estrogen receptor-positive breast cancers and revealed discordant risk of recurrence by using different regions from the same tumors, suggesting that a single biopsy could either under- or over-estimate recurrence risks of breast cancer due to the intra-tumor heterogeneity. To the best of our knowledge, comprehensive gene expression intra-tumor heterogeneity is not well defined in any cancer types. Here, we present our analyses of gene expression profiles of 35 spatially separated regions from 10 patients with resected non-small cell lung cancer.

\section{Materials and methods}

\section{Multiregion sampling and clinical information}

We collected 35 tumor samples from 10 patients with nonsmall cell lung cancer (three or four regions/tumor) including lung adenocarcinoma $(n=6)$, squamous cell carcinoma $(n=2)$, large-cell carcinoma $(n=1)$, and pleomorphic carcinoma $(n=1)$. Multiregion sampling was done as illustrated in Supplementary Figure 1. Detailed clinical information for every patient, such as tumor size, stage, prognosis, and treatment history, can be found in Supplementary Table 1.

\section{Data preprocessing and normalization}

The gene expression data were generated by using the Affymetrix GeneChip ${ }^{\circledR}$ Human Gene 1.0 ST arrays. The platform contains 33,252 transcript clusters (corresponding to 20,009 unique genes). The $\mathrm{R}$ package, aroma.affymetrix, was used to process the expression measurements. Briefly, the CEL formatted files were loaded first, and then the robust multiarray analysis method was used for data processing and background adjustment. The backgroundcorrected measurements were then logarithm transformed (base 2) and quantile normalization was applied.

\section{Gene annotation to transcript cluster}

Once the data were processed, we updated the annotation based on the most recent annotation file (HuGene-1_0-stv1.na36.hg19.transcript.csv, 7/6/16) obtained from the NetAff ${ }^{\mathrm{TM}}$ Analysis Center of Affymetrix, and annotated transcript clusters with NCBI RefSeq genes. For a cluster with multiple unique gene symbols, we aligned the sequence of the cluster with each of the candidate genes using bl2seq (BLAST 2 Sequences) in BLAST package (v2.2.12). A gene with the highest bit-score was considered as the gene for the cluster. If multiple genes show the same best score for a cluster, we assigned them all to the cluster.

\section{Unique gene-level expression profiling}

If a gene is assigned with only one transcript cluster, the cluster represents the expression level of the gene. If multiple transcript clusters point to the same gene, we first calculated Pearson correlation coefficient between them and took the average if they are correlated $(r>0.7)$. Otherwise, individual sequences from the clusters were compared with the gene sequence with bl2seq, and the best matching cluster was selected to represent expression level for the gene. If there are multiple transcript clusters reaching the same best score, we selected one with the highest average expression level across all samples.

\section{Molecular subtyping and epithelial-mesenchymal transition score calculation}

To calculate molecular subtype scores, we obtained subtype predictor centroids for lung adenocarcinoma and squamous cell carcinoma [11, 12]. For lung adenocarcinoma, we renamed subtypes as proposed by The Cancer Genome Atlas [13]: bronchioid to terminal respiratory unit, magnoid to proximal proliferative, and squamoid to proximal inflammatory. Molecular subtype scores were calculated with the method previously used [14]. Briefly, we used the following procedure: (i) extracting expression values of the genes overlapped with the gene lists of subtype centroids, (ii) mean centering within the specific histological group (adenocarcinoma or squamous), (iii) averaging the expression values if there are multiple probes indicating the same gene, (iv) calculating Spearman rank correlation between values of a given sample and centroids, and (v) selecting the subtype showing the highest correlation coefficient with $p$ 


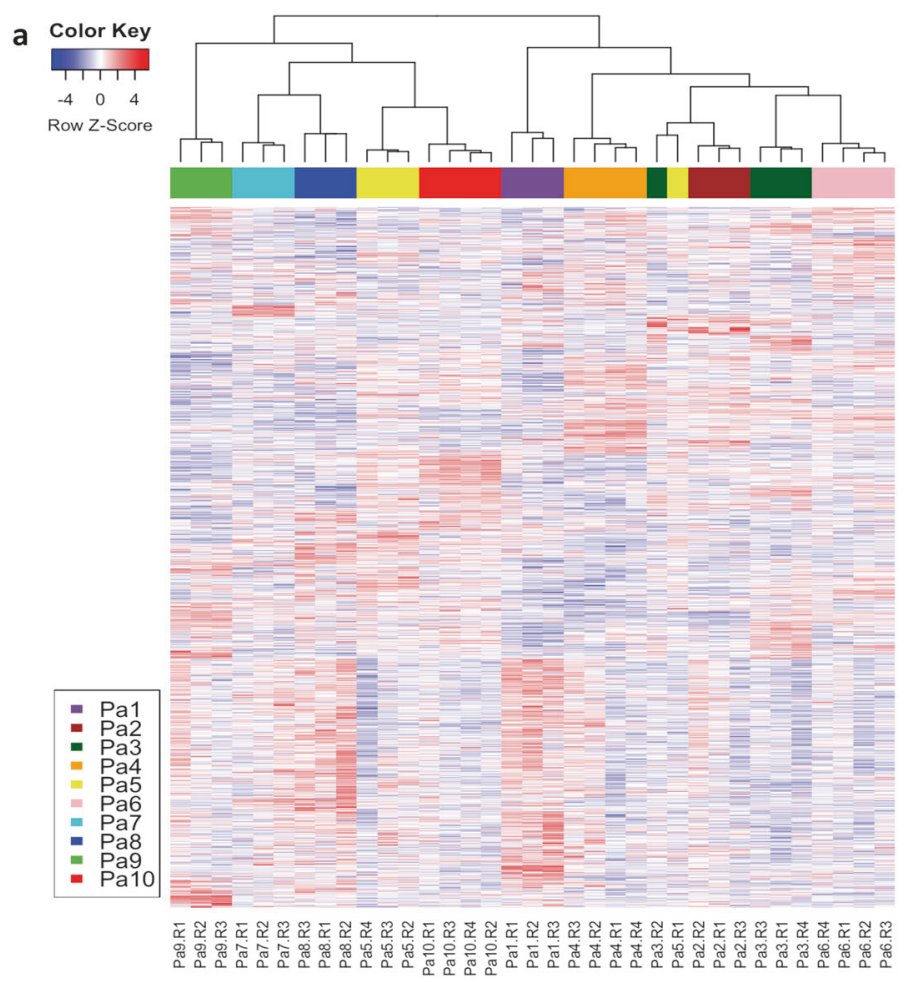

b

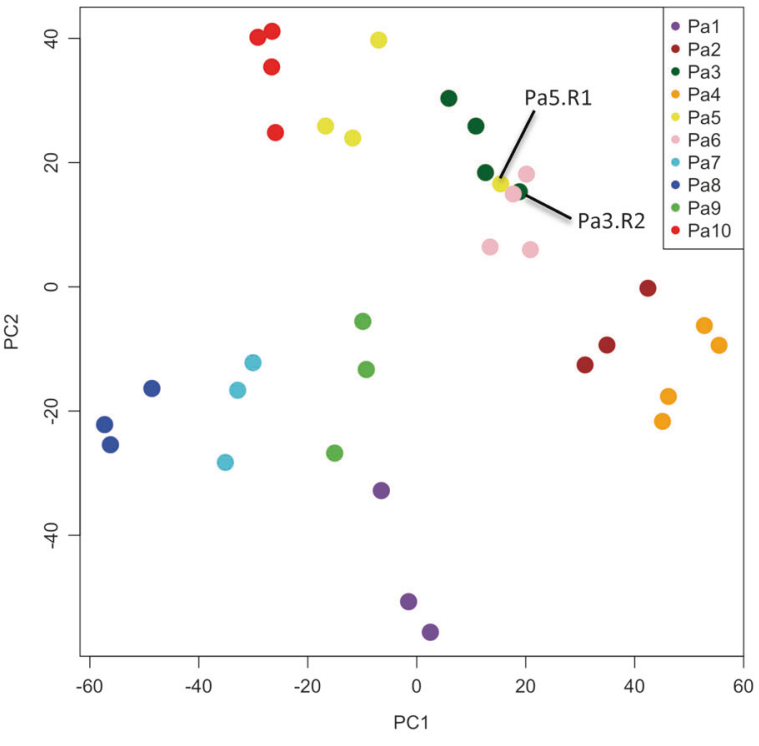

Fig. 1 a Heat map generated by unsupervised hierarchical clustering with all transcript clusters $(n=21,052)$. The 1-correlation distance and ward.D2 linkage were used for clustering. The rows and columns represent transcript clusters and tumor samples, respectively. Samples from the same patients were illustrated in the same colors below the

column dendrogram. b Principal component analysis plot with the two dimensions (PC1 and PC2) along which the data are the largest spread. Samples from the same patients were shown in the same colors. Two samples (Pa3.R2 and Pa5.R1), which clustered with tumor samples from other patients, are indicated

value $<0.01$ as a predicted molecular subtype. We did not calculate subtype scores for large-cell and pleomorphic carcinoma samples.

For epithelial-mesenchymal transition score calculation, we used the 76-gene epithelial-mesenchymal transition signature panel [15] with the average-based method introduced previously [16]. First, expression values of each gene were standardized by the mean and standard deviation. For each sample, an epithelial-mesenchymal transition score was calculated as the average expression values for mesenchymal genes minus the average expression values for epithelial genes.

\section{Prognostic gene signature analysis}

We queried published studies on mRNA microarray-based prognostic signatures for non-small cell lung cancer [17] and selected two frequently cited prognostic signatures for further analyses [18, 19]. The first signature consisting of six genes [18] was originally developed using RT-PCR and validated on four independent public microarray data sets. For each of the six genes, expression values were subtracted by the median of the gene. We skipped housekeeping gene normalization, which was done for the RT-PCR data in the original paper. Euclidean distances to the training cluster

medians (high- or low-risk cluster provided by the original paper) were used to predict prognostic subgroups. The prediction was performed only when the ratio of two distances to the training cluster medians is less than 0.9. The second signature consisting of 15 genes [19] uses supervised principal component analysis to calculate risk scores. For each of the 15 genes, expression values were transformed to $Z$-scores by centering to the mean and scaling to the standard deviation of the gene. In the original paper, the risk scores of four independent published microarray data sets for validation were dichotomized at -0.1 , the median risk score determined in the training data set. With the same principal component analysis rotation matrix and Cox regression coefficients, we derived risk scores for our samples and classified them based on the same cutoff -0.1 .

\section{Calculation of scores for innate anti-PD-1 resistance content signatures}

We used the gene set variation analysis program with default settings to calculate the enrichment scores for the 22 innate anti-PD-1 resistance content signatures reported by the previous publication [20]. The definition files of these signatures were downloaded either from the Molecular Signature Database (MSigDB) [21] or the supplementary 
Fig. 2 Heat maps for adenocarcinoma subtype scores (top heat map) and for squamous cell carcinoma subtype scores (bottom heat map). The predicted molecular subtypes are shown with corresponding abbreviations (a "dot" represents no statistically significant molecular subtype)
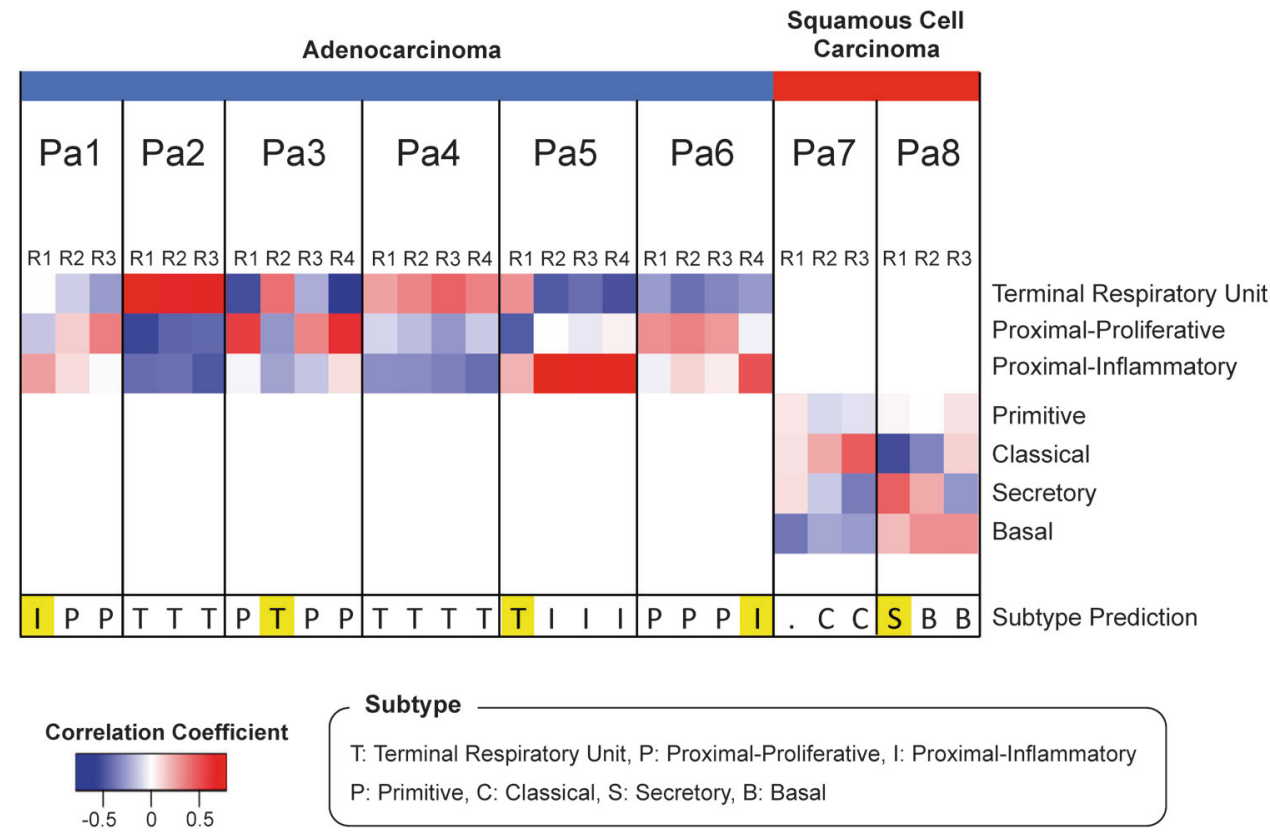

file of the publication. For each signature, the enrichment scores across all samples were converted to $Z$-scores. To compute the innate anti-PD-1 resistance "co-enrichment" score for a given sample, we averaged $Z$-scores for the sample across the 22 innate anti-PD-1 resistance content signatures.

\section{Impact of tumor microenvironment on gene expression heterogeneity}

Using a gene expression-based algorithm ESTIMATE [22], we calculated tumor microenvironment scores (stromal score, immune score, and corresponding tumor purity) for each sample. Then, we correlated them with epithelial-mesenchymal transition, innate anti-PD-1 resistance, and lung adenocarcinoma subtype scores. Overall gene expression profiles were represented by the first principal components and correlated with the tumor microenvironment scores. Pearson correlation coefficient was used to measure the degree of correlation.

\section{Results}

\section{Gene expression heterogeneity between and within non-small cell lung cancers}

To determine the variation of gene expression patterns between different regions of the individual tumors and across the different tumors, we performed unsupervised hierarchical clustering. When comparing all samples across the different patients, different tumor regions from the same patients clustered together for all patients except two tumor samples Pa3.R2 and Pa5.R1, which clustered with tumor samples from other patients (Fig. 1a). The principal component analysis also showed a similar pattern with the two tumor samples mixing with tumors from different patients (intraclass correlation coefficient of the first principal component: $0.96,95 \%$ CI $(0.9,0.98)$, Fig. 1b). These results suggest marked inter-individual gene expression heterogeneity and to a less extent intra-tumor heterogeneity. However, certain tumors may have high level of gene expression intra-tumor heterogeneity that is comparable to the heterogeneity between different tumors.

\section{Varied molecular subtypes within the same tumors}

Molecular subtyping has become a critical component of precision oncology and gene expression profiling has been used for molecular subtyping in many types of cancers including breast cancer [23], glioblastoma [24], colorectal cancer [25], and lung cancer [11-13, 26]. Lung adenocarcinomas can be classified into terminal respiratory unit, proximal proliferative, and the proximal inflammatory subtypes [13], while lung squamous cell carcinomas can be classified into classical, basal, secretory, and primitive subtypes [11]. In the previous studies, the molecular subtyping has been based on a single tumor sample. Since we observed evidence of gene expression intra-tumor heterogeneity, we next investigated whether the molecular subtype is concordant between different tumor regions within the same tumors. As shown in Fig. 2 and Supplementary Table 2, heterogeneous molecular subtype scores were observed between different tumor regions within the same 


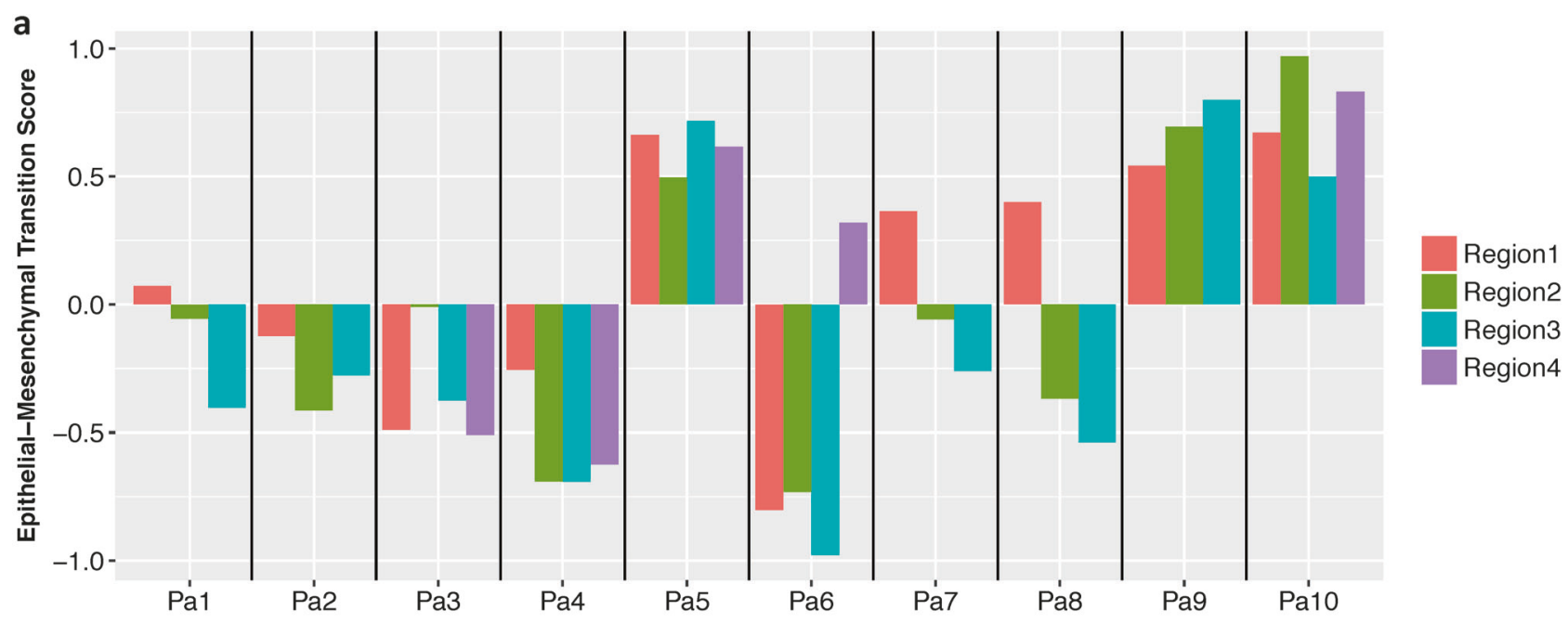

b
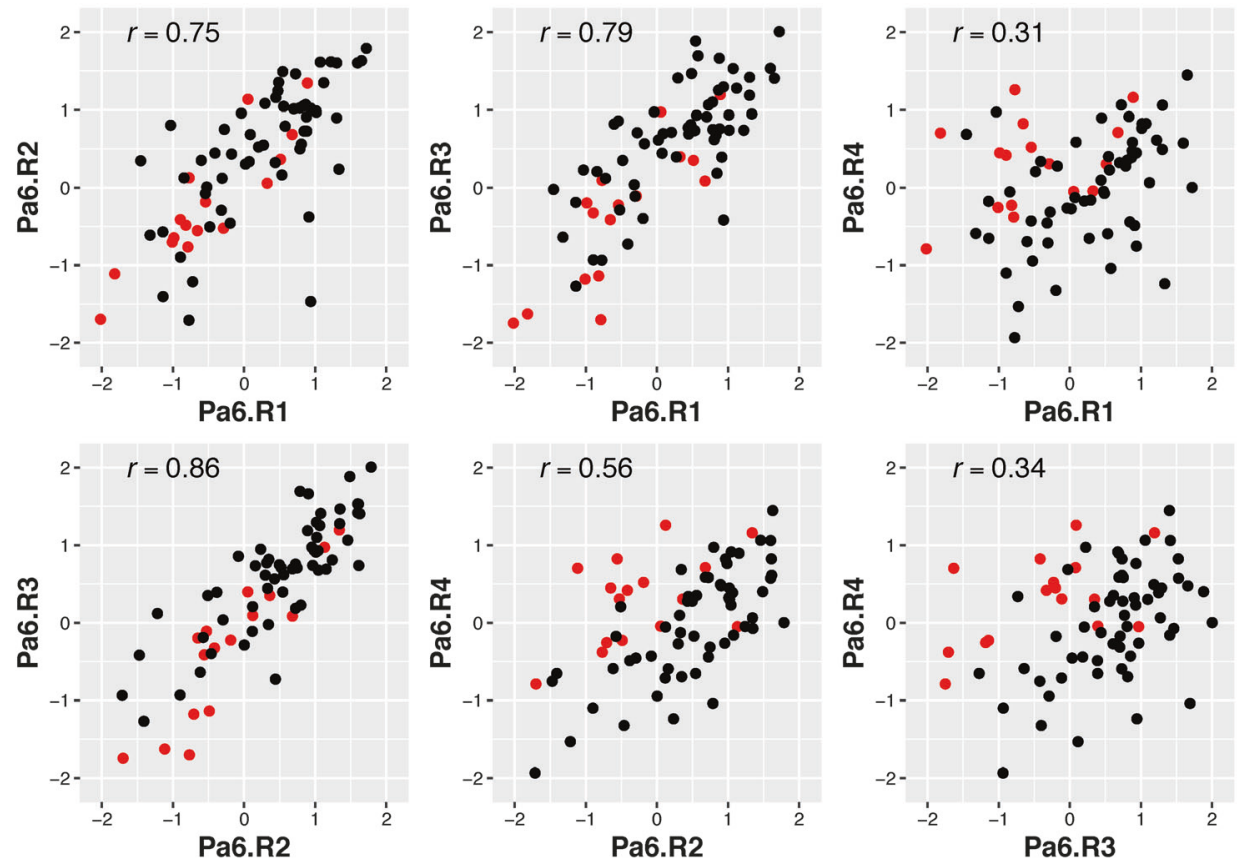

- Epithelial

- Mesenchymal

Fig. 3 a Epithelial-mesenchymal transition scores for all tumor samples. Pa1 to Pa6: adenocarcinoma, Pa7 and Pa8: squamous cell carcinoma, Pa9: large-cell carcinoma and Pa10: pleomorphic carcinoma.

b All pairwise epithelial-mesenchymal transition-related gene expression correlations for Pa6. The Pearson correlation coefficient is shown within each plot

tumors. For example, Pa1.R1 had the highest score for proximal inflammatory subtype, while the other two tumor regions of $\mathrm{Pa} 1$ demonstrated the highest scores for proximal proliferative subtype. In Pa3, terminal respiratory unit is the predicted subtype in Pa3.R2 while all three other samples from $\mathrm{Pa} 3$ are predicted to be proximal proliferative subtype.

\section{Varied prognostic gene signatures within the same tumors}

Numerous gene expression-based profiles of non-small cell lung cancer have been generated over the past 20 years and many diagnostic and prognostic gene signatures have been proposed. Given the substantial gene expression intra-tumor heterogeneity observed in our cohort, we next investigated whether different tumor regions would show different gene signatures that are associated with prognosis of lung cancer patients.

The first signature we looked at was epithelial-mesenchymal transition. Epithelial-mesenchymal transition is a biological process, by which epithelial cells lose cell adhesion molecules and become more like mesenchymal cells with high migratory and invasive capabilities. Epithelial-mesenchymal transition was reported to be a potential prognostic and predictive marker in patients 
a

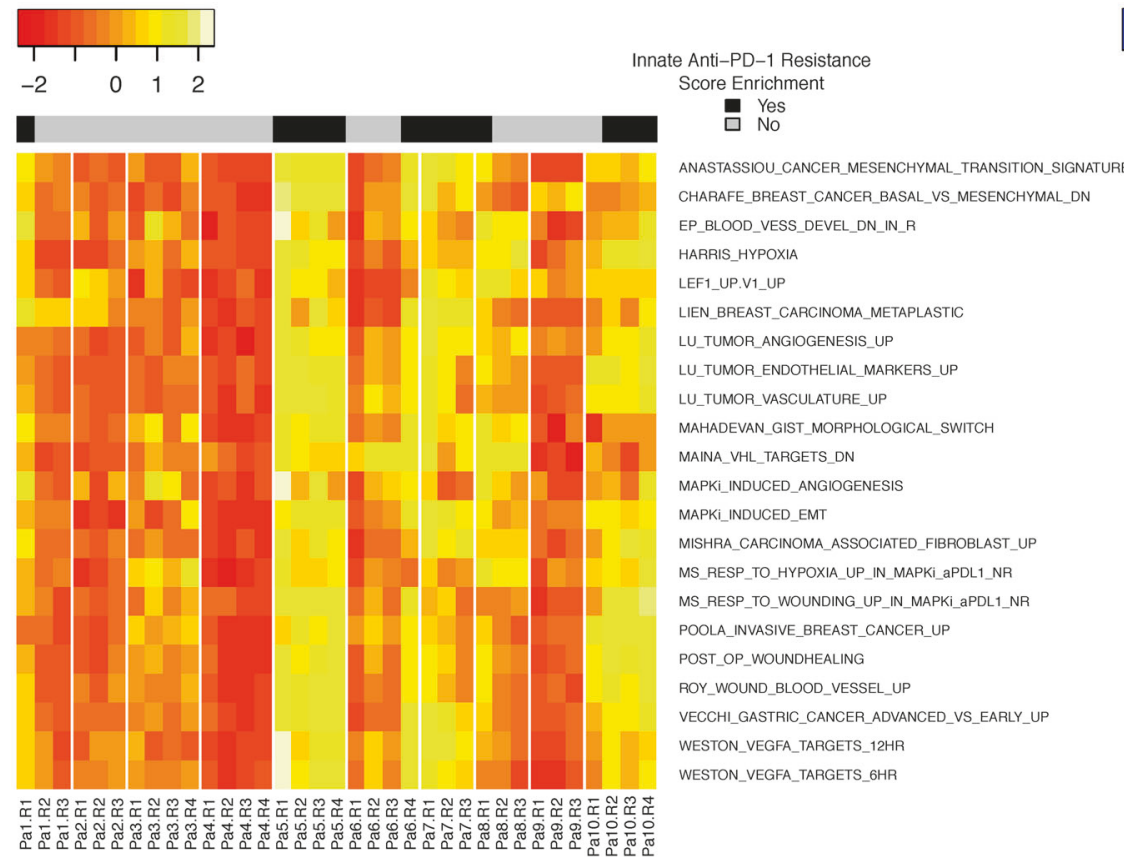

b

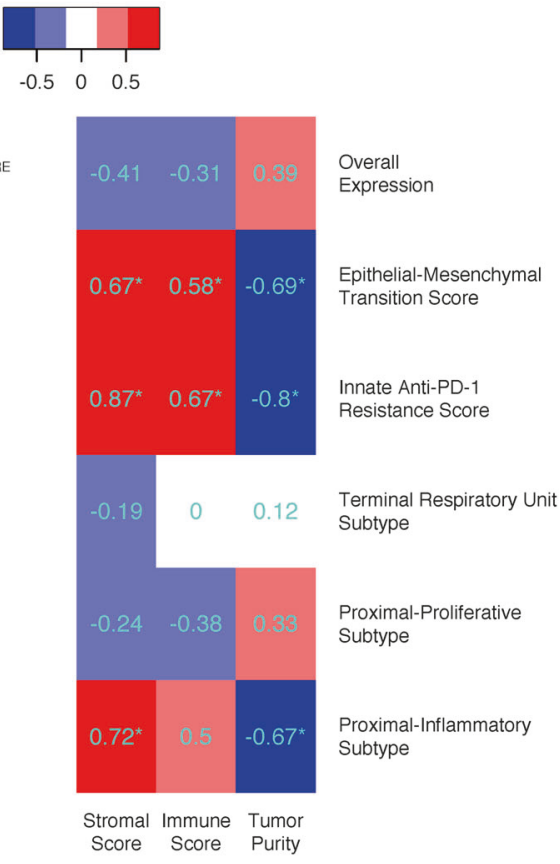

Fig. 4 a Gene set variation analysis enrichment scores for 22 innate anti-PD-1 resistance content signatures were calculated for each sample. The rows represent signatures and the columns represent samples. Innate anti-PD-1 resistance co-enrichment status was determined by the innate anti-PD-1 resistance co-enrichment score cutoff
0.35 and shown on top of the heat map. b Correlation of tumor microenvironment scores with various gene expression signatures examined in this study. Pearson correlation coefficients are shown and the ones with $p$ value $<0.01$ are marked with an asterisk with lung cancer [27-30]. Using our previously developed 76-gene epithelial-mesenchymal transition signature panel [15], we computed an epithelial-mesenchymal transition score for each sample (Fig. 3a and Supplementary Table 3). Consistent with previous studies [31, 32], different tumors demonstrated different epithelial-mesenchymal transition scores and the poorly differentiated large-cell carcinoma, pleomorphic carcinoma, and squamous cell carcinomas showed higher (i.e., more mesenchymal) scores than adenocarcinomas ( $p$ value: 0.003). Different regions of the same tumors demonstrated similar epithelial-mesenchymal transition scores for the most part (intraclass correlation coefficient of epithelial-mesenchymal transition score: 0.71 , $95 \%$ CI $(0.43,0.90))$; however, considerable intratumor heterogeneity of epithelial-mesenchymal transition scores were observed in some tumors. For example, within tumor Pa6, only Pa6.R4 clearly demonstrated overexpression of mesenchymal-specific genes and corresponding high epithelial-mesenchymal transition score, while all the other three tumor regions showed very low epithelial-mesenchymal transition scores (Fig. 3a). In addition, although the expression levels of epithelial-mesenchymal transition-related genes were mostly correlated between different regions within the same tumors, the correlation was weak for many tumor pairs further suggesting the spatially heterogeneous expression of epithelial-mesenchymal transition-related genes (Fig. 3b and Supplementary Figure 2).

Next, we selected two other frequently cited mRNA microarray-based prognostic signatures [18, 19] and calculated the risk scores in each tumor region. As expected, the risk score varied between different tumor regions within the same tumors (Supplementary Table 4). Interestingly, when every tumor region was classified into high- and low-risk prognostic subgroups based on the same criteria in the original studies, discordant prognostication derived from different tumor regions within the same tumor was observed in 1 of the 10 patients using the 6-gene signature and in 7 of the 10 patients using the 15-gene signature, respectively. These results suggest that gene expression-based prognostic signature from a single biopsy could either under- or overestimate the risk of recurrence of patients with non-small cell lung cancer due to the gene expression intra-tumor heterogeneity.

\section{Varied innate anti-PD-1 resistance signatures within the same tumor}

Recent studies have shown that gene expression-based molecular subtypes were associated with response to immune checkpoint inhibition in bladder cancer [33] and melanoma [20]. Hugo et al. reported innate anti-PD-1 
resistance transcriptomic signature in melanoma and other cancers including lung cancer [20]. Using the same innate anti-PD-1 resistance signature, we characterized our tumor samples (Fig. 4a). For a given sample, we computed the innate anti-PD-1 resistance co-enrichment score to measure the level of co-enrichment of the 22 innate anti-PD-1 resistance content signatures (Supplementary Table 5). In general, innate anti-PD-1 resistance co-enrichment score was similar within the same tumor (intraclass correlation coefficient of innate anti-PD-1 resistance co-enrichment score: 0.77 , 95\% CI $(0.52,0.93))$. However, spatial heterogeneity was observed in multiple tumors. When we dichotomized samples by the innate anti-PD-1 resistance co-enrichment score cutoff $(0.35)$ that was used in the original literature, 4 in 10 patients harbored at least one discordant tumor region. For example, in tumor Pa6, Pa6.R4 is considered as an anti-PD-1-resistant tumor by showing the high innate anti-PD-1 resistance co-enrichment score of 0.94. However, the other three tumor regions (Pa6.R1, Pa6. $\mathrm{R} 2$, and Pa6.R3) showed low innate anti-PD-1 resistance co-enrichment scores of $-0.94,0.06$, and -0.29 , respectively. Our data suggest that the intra-tumor heterogeneity may need to be taken into consideration if gene expressionassociated signatures were to be used as biomarkers for immune checkpoint blockade therapy.

\section{Varied tumor microenvironment}

Cancers are not homogenous masses of malignant cells. Many other cells are recruited to the tumor tissue including fibroblasts, blood and lymphatic vessel endothelial cells, and infiltrating immune cells, which constitute the tumor microenvironment. Each cell type may have distinct gene expression profile of its own and the overall gene expression profile of a given tumor sample reflects the amount of each cell type and the interactions between cancer cells and non-transformed cells in that tumor microenvironment. To understand the impact of tumor microenvironment on gene expression intra-tumor heterogeneity, we inferred the proportions of infiltrating stromal and immune cells for each tumor region using a gene expression-based algorithm ESTIMATE [22] and correlated tumor microenvironment scores with the gene expression signatures examined in this study. Our results showed that different tumor regions have different proportions of cancer cells, infiltrating stromal and immune cells (Supplementary Table 6). As shown in Fig. 4b and Supplementary Figure 3a, the overall gene expression variation was only weakly correlated with stromal score, immune score, or tumor purity with Pearson correlation coefficient of $-0.41,-0.31$, and 0.39 , respectively. However, we observed high level of correlation (absolute Pearson correlation coefficient: 0.58-0.87) between epithelial-mesenchymal transition/innate anti-PD-1 resistance score and stromal/immune score (Fig. 4b and Supplementary Figure 3b-c). Among the lung adenocarcinoma molecular subtypes, proximal inflammatory subtype was positively correlated with stromal and immune scores (Fig. 4b and Supplementary Figure 3f).

\section{Discussion}

Using an approach of multiregion sampling and comprehensive gene expression analysis of 10 non-small cell lung cancers, we demonstrated clear evidence of gene expression intra-tumor heterogeneity in all tumors studied. Two tumor samples showed substantial level of global intra-tumor heterogeneity comparable to heterogeneity between different patients (Fig. 1). In addition to the global gene expression profile, substantial intra-tumor heterogeneity was also observed for molecular subtypes and various clinically relevant gene expression signatures including epithelial-mesenchymal transition signature, a recently reported immune therapy response signature and two frequently cited prognostic signatures (Figs. 2-4 and Supplementary Tables 2-5). Interestingly, global intra-tumor heterogeneity did not always correlate with intra-tumor heterogeneity observed in the signatures with selected genes.

Programmed cell death protein 1 (PD-1) immune checkpoint blockade has shown unprecedented durable clinical responses in patients with various cancer types including metastatic non-small cell lung cancer [34]; however, the response rate is suboptimal [35, 36]. There are currently no reliable biomarkers to predict response to immune checkpoint blockade. Expression of programmed death-ligand 1 (PD-L1) protein was reported to be predictive of benefit from immune checkpoint blockade treatment, but robust responses seen in patients with low PD-L1 argues against its value as an exclusionary predictive biomarker [37]. Gene expression-based signatures have been recently reported to correlate with response to immune checkpoint inhibition in various cancer types and proposed as potential predictive markers [20, 33]. Using a recently published innate anti-PD-1 resistance signature analysis, our results showed 4 out of the 10 patients harbored substantially different innate anti-PD-1 resistance scores in different tumor regions within the same tumors that may lead to discordant prediction of response to anti-PD-1 checkpoint inhibition. When correlating to the tumor microenvironment, innate anti-PD-1 resistance score was found to correlate negatively with tumor purity, and positively with immune score and stromal score, which is as expected because it is well known that tumor infiltrating lymphocyte profile, particularly CD8 $+\mathrm{T}$ cell infiltrate is associated with response to anti-PD-1 therapy [38]. What is 
interesting is that the correlation of innate anti-PD-1 resistance score with stromal score was higher than that with the immune score (correlation coefficient of 0.87 versus 0.67 ), indicating that in addition to immune cells, other stromal cells may also contribute to impact on the anti-PD-1 response signature.

Cancer tissues are composed of both cancer cells and non-cancerous stromal cells that form the tumor microenvironment. Our data demonstrated heterogeneous tumor microenvironments in spatially separated tumor regions within the same tumor (Supplementary Table 6). This heterogeneous tumor microenvironment is closely correlated to some characteristics of tumor represented by the gene expression signatures explored in this study (Supplementary Figure 3). However, these correlations were only marginal at overall gene expression profile. In addition, different tumor regions from the same patients almost always clustered together in spite of different tumor purity, stromal or immune scores (Fig. 1). Taken together, our data suggest that the gene expression intra-tumor heterogeneity observed in this study may be attributed to the different tumor microenvironments as well as spatial difference in gene expression profiles of lung cancer cells.

Many prognostic gene signatures have been developed by diverse analytical techniques. Commercially available and clinically applicable prognostic tests like Pervenio ${ }^{\mathrm{TM}}$ Lung RS [39] are often based on small prognostic and predictive gene sets with RT-PCR protocol. Future studies include the investigation on the intra-tumor heterogeneity of those signatures with the same techniques by which they were developed. Moreover, higher-resolution technologies, such as RNA sequencing on a larger cohort of patients, ideally supported by genomics and epigenetic data will help us to better understand the gene expression intra-tumor heterogeneity in lung cancer and its impact on cancer biology and clinical outcomes.

In summary, our study is the first study, to the best of our knowledge, on comprehensive gene expression intra-tumor heterogeneity of any cancer type. We observed clear evidence of gene expression intra-tumor heterogeneity from all tumors in this cohort for both the overall gene expression profile and the expression signatures with selected genes. Since many of these gene expression signatures have been reported to be associated with clinical outcomes of lung cancers and proposed as potential prognostic and/or predictive biomarkers, our results suggest that gene expression intra-tumor heterogeneity should be taken into consideration when evaluating gene expression-based biomarkers.

Acknowledgements This work was supported by MD Anderson Lung cancer Moon Shot Program, MD Anderson Physician Scientist Program, Conquer Cancer Foundation Young Investigator Award, Khalifa Scholar Award, the Cancer Prevention and Research Institute of Texas (R120501), the Cancer Prevention and Research Institute of Texas
Multi-Investigator Research Award grant (RP160668), the University of Texas (UT) Systems Stars Award (PS100149), the Welch Foundation Robert A. Welch Distinguished University Chair Award (G0040), Department of Defense PROSPECT grant (W81XWH-07-10306), the UT Lung Specialized Programs of Research Excellence grant (P50CA70907), and T.J. Martell Foundation.

\section{Compliance with ethical standards}

Conflict of interest The authors declare that they have no conflict of interest.

\section{References}

1. The Cancer Genome Atlas - National Cancer Institute. http://ca ncergenome.nih.gov. Accessed 15th July 2016.

2. McGranahan N, Swanton C. Biological and therapeutic impact of intratumor heterogeneity in cancer evolution. Cancer Cell. 2015;27:15-26.

3. Gerlinger M, Rowan AJ, Horswell S, et al. Intratumor heterogeneity and branched evolution revealed by multiregion sequencing. N Engl J Med. 2012;366:883-92.

4. Gerlinger M, Horswell S, Larkin J, et al. Genomic architecture and evolution of clear cell renal cell carcinomas defined by multiregion sequencing. Nat Genet. 2014;46:225-33.

5. Zhang J, Fujimoto J, Zhang J, et al. Intratumor heterogeneity in localized lung adenocarcinomas delineated by multiregion sequencing. Science. 2014;346:256-9.

6. de Bruin EC, McGranahan N, Mitter R, et al. Spatial and temporal diversity in genomic instability processes defines lung cancer evolution. Science. 2014;346:251-6.

7. Pertschuk LP, Axiotis CA, Feldman JG, et al. Marked intratumoral heterogeneity of the proto-oncogene Her-2/neu determined by three different detection systems. Breast J. 1999;5:369-74.

8. Chung GG, Zerkowski MP, Ghosh S, Camp RL, Rimm DL. Quantitative analysis of estrogen receptor heterogeneity in breast cancer. Lab Invest. 2007;87:662-9.

9. Yang J, Luo H, Li Y, et al. Intratumoral heterogeneity determines discordant results of diagnostic tests for human epidermal growth factor receptor (HER) 2 in gastric cancer specimens. Cell Biochem Biophys. 2012;62:221-8.

10. Gyanchandani R, Lin Y, Lin HM, et al. Intratumor heterogeneity affects gene expression profile test prognostic risk stratification in early breast cancer. Clin Cancer Res. 2016;22:5362-9.

11. Wilkerson MD, Yin X, Hoadley KA, et al. Lung squamous cell carcinoma mRNA expression subtypes are reproducible, clinically important, and correspond to normal cell types. Clin Cancer Res. 2010;16:4864-75.

12. Wilkerson MD, Yin X, Walter V, et al. Differential pathogenesis of lung adenocarcinoma subtypes involving sequence mutations, copy number, chromosomal instability, and methylation. PLoS ONE. 2012;7:e36530.

13. Cancer Genome Atlas Research Network. Comprehensive molecular profiling of lung adenocarcinoma. Nature. 2014;511: 543-50.

14. Ringner M, Jonsson G, Staaf J. Prognostic and chemotherapy predictive value of gene-expression phenotypes in primary lung adenocarcinoma. Clin Cancer Res. 2016;22:218-29.

15. Byers LA, Diao L, Wang J, et al. An epithelial-mesenchymal transition gene signature predicts resistance to EGFR and PI3K inhibitors and identifies Axl as a therapeutic target for overcoming EGFR inhibitor resistance. Clin Cancer Res. 2013;19:279-90.

16. Chen L, Gibbons DL, Goswami S, et al. Metastasis is regulated via microRNA-200/ZEB1 axis control of tumour cell PD-L1 
expression and intratumoral immunosuppression. Nat Commun. 2014;5:5241.

17. Kuner R. Lung cancer gene signatures and clinical perspectives. Microarrays. 2013;2:318-39.

18. Boutros PC, Lau SK, Pintilie M, et al. Prognostic gene signatures for non-small-cell lung cancer. Proc Natl Acad Sci USA. 2009;106:2824-8.

19. Zhu CQ, Ding K, Strumpf D, et al. Prognostic and predictive gene signature for adjuvant chemotherapy in resected non-small-cell lung cancer. J Clin Oncol. 2010;28:4417-24.

20. Hugo W, Zaretsky JM, Sun L, et al. Genomic and transcriptomic features of response to anti-PD-1 therapy in metastatic melanoma. Cell. 2016;165:35-44.

21. Subramanian A, Tamayo P, Mootha VK, et al. Gene set enrichment analysis: a knowledge-based approach for interpreting genome-wide expression profiles. Proc Natl Acad Sci USA. 2005; 102:15545-50.

22. Yoshihara K, Shahmoradgoli M, Martinez E, et al. Inferring tumour purity and stromal and immune cell admixture from expression data. Nat Commun. 2013;4:2612.

23. Perou CM, Sorlie T, Eisen MB, et al. Molecular portraits of human breast tumours. Nature. 2000;406:747-52.

24. Verhaak RG, Hoadley KA, Purdom E, et al. Integrated genomic analysis identifies clinically relevant subtypes of glioblastoma characterized by abnormalities in PDGFRA, IDH1, EGFR, and NF1. Cancer Cell. 2010;17:98-110.

25. Guinney J, Dienstmann R, Wang $X$, et al. The consensus molecular subtypes of colorectal cancer. Nat Med. 2015;21: 1350-6.

26. Cancer Genome Atlas Research Network. Comprehensive genomic characterization of squamous cell lung cancers. Nature. 2012;489:519-25.

27. Thomson S, Buck E, Petti F, et al. Epithelial to mesenchymal transition is a determinant of sensitivity of non-small-cell lung carcinoma cell lines and xenografts to epidermal growth factor receptor inhibition. Cancer Res. 2005;65:9455-62.

28. Yauch RL, Januario T, Eberhard DA, et al. Epithelial versus mesenchymal phenotype determines in vitro sensitivity and predicts clinical activity of erlotinib in lung cancer patients. Clin Cancer Res. 2005;11:8686-98.

29. Frederick BA, Helfrich BA, Coldren CD, et al. Epithelial to mesenchymal transition predicts gefitinib resistance in cell lines of head and neck squamous cell carcinoma and non-small cell lung carcinoma. Mol Cancer Ther. 2007;6:1683-91.

30. Papadimitrakopoulou V, Lee JJ, Wistuba, II, et al. The BATTLE2 study: a biomarker-integrated targeted therapy study in previously treated patients with advanced non-small-cell lung cancer. J Clin Oncol. 2016;34:3638-47.

31. Nitsche K, Gunther B, Katenkamp D, Petersen I. Thoracic neoplasms at the Jena reference center for soft tissue tumors. J Cancer Res Clin Oncol. 2012;138:415-24.

32. Matsubara D, Kishaba Y, Yoshimoto T, et al. Immunohistochemical analysis of the expression of E-cadherin and ZEB1 in non-small cell lung cancer. Pathol Int. 2014;64:560-8.

33. Rosenberg JE, Hoffman-Censits J, Powles T, et al. Atezolizumab in patients with locally advanced and metastatic urothelial carcinoma who have progressed following treatment with platinumbased chemotherapy: a single-arm, multicentre, phase 2 trial. Lancet. 2016;387:1909-20.

34. Pardoll DM. The blockade of immune checkpoints in cancer immunotherapy. Nat Rev Cancer. 2012;12:252-64.

35. Brahmer JR, Tykodi SS, Chow LQ, et al. Safety and activity of anti-PD-L1 antibody in patients with advanced cancer. N Engl J Med. 2012;366:2455-65.

36. Topalian SL, Hodi FS, Brahmer JR, et al. Safety, activity, and immune correlates of anti-PD-1 antibody in cancer. N Engl J Med. 2012;366:2443-54.

37. Patel SP, Kurzrock R. PD-L1 expression as a predictive biomarker in cancer immunotherapy. Mol Cancer Ther. 2015;14:847-56.

38. Tumeh PC, Harview CL, Yearley JH, et al. PD-1 blockade induces responses by inhibiting adaptive immune resistance. Nature. 2014;515:568-71.

39. Kratz JR, He J, Van Den Eeden SK, et al. A practical molecular assay to predict survival in resected non-squamous, non-small-cell lung cancer: development and international validation studies. Lancet. 2012;379:823-32. 